\title{
Diseño de herramientas que fomentan el aprendizaje de matemáticas con ayuda de Mathematica 10
}

\section{Design of Tools that Promote Math Learning by Using Mathematica 10}

\author{
Camilo Andrés Ramírez ${ }^{\star}$ \\ Facultad de Ingeniería y Ciencias Básicas, \\ Institución Universitaria Politécnico Grancolombiano, \\ Bogotá, Colombia.
}

FeCha DE ENTREGa: 13 DE ENERO DE 2015

FECHA DE EVALUACIÓN: 16 DE MARZO DE 2015

FECHA DE APROBACIÓN: 24 DE ABRIL DE 2015

\begin{abstract}
Resumen En este artículo se presenta el desarrollo y avances del proyecto "Diseño e implementación de objetos virtuales y dinámicos en el aprendizaje de matemáticas" correspondiente a la elaboración de una serie de programas con el objeto de ampliar las herramientas que apoyan a estudiantes y docentes, y orientan el curso de Matemáticas de la Institución Universitaria Politécnico Grancolombiano. En primera instancia se muestra el marco teórico que fundamenta el desarrollo del proyecto identificando las problemáticas y posibles vías de acción, seguido de esto se da una breve introducción al programa trabajado y los productos esperados. Finalmente se muestra el desarrollo y algunos avances en el proceso de implementación donde las evidencias sugieren que con estas herramientas se logra acercar un poco al estudiante (no a todos) a la comprensión de los conceptos matemáticos.
\end{abstract}

Abstract This article presents the development and progress of the project "Design and Implementation of Virtual and Dynamic Objects when Learning Math". This project is part of a series of programs that search for enhancing of tools that support math students and teachers at

* Docente de matemáticas con énfasis en Tecnología de la Universidad Pedagógica Nacional y Magister en Matemática Aplicada de la Universidad Nacional de Colombia, con una formación pedagógica y disciplinar para el desempeño en la educación matemática en básica, media y universitaria. Conocimientos avanzados en el área de sistemas, programación, software matemático y manejo de plataformas de aprendizaje virtual. Amplia experiencia en la planeación, organización y estructuración de congresos y encuentros de educación matemática. Actualmente es docente de planta del Politécnico Grancolombiano en donde se especializa en la tutoría, administración y logística de módulos del área de matemáticas en la modalidad de educación virtual. caramirezs@poligran.edu.co 
Politécnico Grancolombiano University Institution. Initially, we showed the theoretical framework that is the foundation of the project by identifying the problem and possible ways to follow. After this, we introduced the program and the expected products. Finally, we presented the development and the improvement of the implementation process. Here, the evidence suggests that it is possible to make most of students understand mathematical concepts by using these tools.

Palabras Clave: aprendizaje significativo, interactividad, matemáticas, educación matemática, applet, apoyo académico.

Keywords: meaningful learning, interactivity, math, math learning, applet, academic support.

\section{Introducción}

El origen de este proyecto está relacionado con varias problemáticas específicas del curso, aspectos curriculares, sociales y herramientas innovadoras que llevaron a reflexionar sobre la necesidad de justificar y realizar programas que propicien una nueva metodología de clase en el curso de Matemáticas que se imparte en la institución.

El diseño de las herramientas que se muestran en este artículo, se enmarca en un proyecto de investigación más amplio que contempla, además de esta fase, una fase de implementación, una de medición de las herramientas en cursos pilotos y una fase de evaluación de esta propuesta, en contraste a los cursos tradicionales.

Actualmente la Institución cuenta con un curso de Matemáticas I para todos los estudiantes, el cual parte del reconocimiento de las matemáticas como herramienta en la vida cotidiana. Este curso se creó para ofrecer un espacio para que el estudiante confronte, revise y modifique lo que cree que sabe, lo que cree que significa estudiar y lo que cree que significa aprender. El logro fundamental de esta asignatura es brindar experiencias que permitan el aprendizaje comprensivo de conceptos matemáticos, el aprendizaje de mecanismos y estrategias para aprender matemáticas y establecer habilidades básicas de matemáticas.

En lo que sigue (sección 2), se resume el marco teórico y el problema de investigación. En la sección 3 se describe la metodología de trabajo, el programa utilizado para su diseño y la descripción y propiedades de los productos finales. En la sección 4 se muestran los avances en la implementación de los programas en un curso piloto y las primeras experiencias. Actualmente el proyecto está en curso y aún no se tienen resultados evaluativos. En las últimas secciones se analiza la idoneidad de la propuesta basada en los resultados parciales de la investigación, así como también se identifican las limitaciones y mejoras potenciales de esta y se termina con una síntesis de la experiencia.

\section{Marco teórico}

Actualmente se puede afirmar que casi todos los fenómenos físicos y situaciones cotidianas se relacionan con "lo que cambia y lo que varía", y es por esta razón que 
se deben desarrollar competencias necesarias en los estudiantes para "entender y controlar el mundo cambiante en el que vivimos" [8]. El reto de la educación actual debe ser conseguir una enseñanza de las matemáticas cognitivamente eficiente. Las tecnologías y software educativos han permitido dar un gran paso en este reto $[6,9]$.

Las funciones que desempeñan los materiales manipulativos (tangibles o gráfico-textuales) en el aprendizaje de la matemática, se fundamenta en teorías que dan un peso importante a las relaciones entre el lenguaje y el pensamiento, y conceden gran relevancia a los medios de expresión en el estudio de las matemáticas [4].

Así mismo, diversas investigaciones están demostrando que los estudiantes pueden aprender más matemáticas y de manera más profunda con el uso de una tecnología apropiada sin llegar a cometer el error de usarla como sustituto de intuiciones y comprensiones conceptuales. Los recursos tecnológicos se deben usar de manera amplia y responsable con el fin de enriquecer el aprendizaje matemático de los estudiantes [4].

Por lo anterior, se fundamenta que el uso de la tecnología en el aula de clase juega un papel cuya importancia se ha incrementado en los últimos años; específicamente en el aprendizaje de las matemáticas, los instrumentos tecnológicos (como calculadoras graficadoras, tablets, computadoras o software específicos de matemáticas) están permitiendo evaluar nuevamente el currículo porque anteriormente, gran parte (o todo) del peso de la enseñanza estaba en desarrollar competencias operativas y algorítmicas, y se dejaba de lado el desarrollo de competencias comprensivas y conceptuales $[1,3]$.

El desarrollo del pensamiento matemático visto desde el punto de vista de competencias requiere enfocarse de manera diferente en el tratamiento procedimental y brindar a los estudiantes más espacios de acompañamiento en los procesos cognitivos y conceptuales [5]. El uso de la tecnología juega un papel importante en el aprendizaje de las matemáticas y en el aprendizaje procedimental de los conceptos matemáticos [2], actualmente existe un software matemático que permite desarrollar objetos dinámicos que potencien la construcción de conceptos matemáticos en el estudiante.

\subsection{El problema de investigación}

Actualmente los problemas frecuentes en los cursos de matemáticas y en los procesos de enseñanza de esta área, se centran en el gran número de estudiantes y la poca disposición que tienen para afrontar el curso responsablemente. Muchos de los estudiantes que toman alguna asignatura de Ciencias Básicas (matemáticas, cálculo, álgebra lineal, estadística, física, química, etc) llegan con una predisposición negativa a la asignatura; por un lado están los estudiantes que durante todo el curso refuerzan esa disposición negativa y apática, lo cual les impide aprovechar las actividades y herramientas que el curso ofrece; y por otro lado están aquellos estudiantes que encuentran un apoyo fundamental en el docente, las herramientas y actividades del curso para afrontar la problemática y la apatía satisfactoriamente. 
Entre los factores que inciden en el logro de los estudiantes, tanto en matemáticas como en otras áreas, han sido los objetos virtuales y las herramientas informáticas de apoyo; más específicamente, las herramientas informáticas que pueden ajustarse al nivel del estudiante y darles retroalimentación [5,7]. Dicho lo anterior, el problema de investigación consiste en diseñar herramientas tecnológicas que incidan positivamente en la disposición del estudiante a la materia y en el aprendizaje de los conceptos de ésta.

\subsection{La herramienta}

En la comunidad académica existen gran variedad de software y lenguajes de programación que permiten el diseño de programas (por ejemplo Maple 17, Mathematica 10, Matlab, java, $C++$ entre otros), se ha optado por utilizar el programa Mathematica 10 pues este posee un potente lenguaje de programación funcional y la característica de compilar programas en documentos dinámicos y manipulables con una interfaz de usuario cómoda y fácil de trabajar. Así mismo posee un gran y número de funciones matemáticas que facilita la elaboración de los programas específicos de esta área incluso para aquellos que no están familiarizados con algún lenguaje de programación.

Mathematica 10 exporta los programas en el formato cdf (Computable Document Format), y para poder ejecutarlos es necesario descargar el plugin "cdf player" el cual es gratuito y se encuentra disponible para cualquier sistema operativo. Los programas desarrollados son de libre licenciamiento, lo cual permite que cualquier persona pueda tener acceso a estos.

\section{Método}

El desarrollo de la investigación se dividió en dos partes, la primera se enfocó en desarrollar herramientas tecnológicas para el docente en el aula y para el estudiante en los momentos de trabajo individual o grupal que motiven el desarrollo de los conceptos matemáticos abordados en la materia:

- Conjunto numéricos (números naturales, enteros, racionales y reales)

- Ecuaciones

- Inecuaciones

- Función lineal y cuadrática

La segunda parte (aunque aún no se ha comenzado) mide la eficacia de las herramientas mediante la implementación de estas en cursos pilotos, comparando los resultados finales con los cursos convencionales.

Para el desarrollo de la primera parte se comenzó con un estudio bibliográfico acerca de la epistemología, didáctica y problemas de enseñanza de los conceptos y competencias matemáticas abordados en el currículo de la asignatura.

Con estos insumos pedagógicos, se procedió a realizar reuniones semanales con el grupo de trabajo en donde se estudió un concepto matemático (por ejemplo, sistemas numéricos) y se identificaron las principales dificultades y problemáticas 
específicas de los estudiantes de la institución, con estos datos se procedió a trabajar en la creación del programa. El desarrollo de cada programa tuvo una duración de 15 a 20 días, lo cual permitió que en una reunión posterior fuera evaluado por el grupo de trabajo su utilidad y se realizaran las correcciones necesarias.

Iniciando el segundo semestre se brindó con algunos programas que fueron utilizados en el aula de clase, esto ofreció la primera aproximación al estudiante y se pudo trabajar en las correcciones necesarias. Afortunadamente se pudieron aplicar todos los programas, lo cual brindó valiosa información e hizo que algunos de estos se modificaran para la versión final.

Finalizando el año se hará la entrega de los productos finales y se comenzará a organizar la puesta en marcha de la segunda fase.

\section{Desarrollo y experiencia en la implementación}

En total se desarrollaron 14 programas que abordaron los principales conceptos de la materia, así:

1. Números naturales y enteros

2. Números reales

3. Expresiones algebraicas

4. Potenciación

5. Factorización

6. Ecuaciones de primer grado

7. Ecuación cuadrática

8. Ecuación no lineal, no cuadrática

9. Inecuaciones lineales

10. Inecuaciones no lineales

11. Concepto de función

12. Transformaciones de funciones

13. Función lineal

14. Función cuadrática

Todo programa consta de una variedad de ejercicios aleatorios, una ayuda "paso a paso" para que el estudiante se centre en los procesos que generen mayor dificultad y una retroalimentación del desarrollo completo del ejercicio.

A continuación se presentan cuatro programas desarrollados e implementados en un curso piloto de la asignatura de Matemáticas de la Institución Universitaria Politécnico Grancolombiano.

\subsection{Ecuación no lineal, no cuadrática}

A comienzo del segundo corte, se comenzó a trabajar con problemas cuya solución se abordó con el uso de ecuaciones. El objetivo era que el estudiante interiorizara los procedimientos algorítmicos para enfrentarse a cualquier tipo de ecuación y pudiera encontrar, de ser posible, las respectivas soluciones. Para ello, se 
desarrollaron tres programas: uno para ecuaciones lineales, otro para ecuaciones cuadráticas y el último para ecuaciones no lineales y no cuadráticas. En la figura 1 se muestra la interfaz gráfica del último programa.

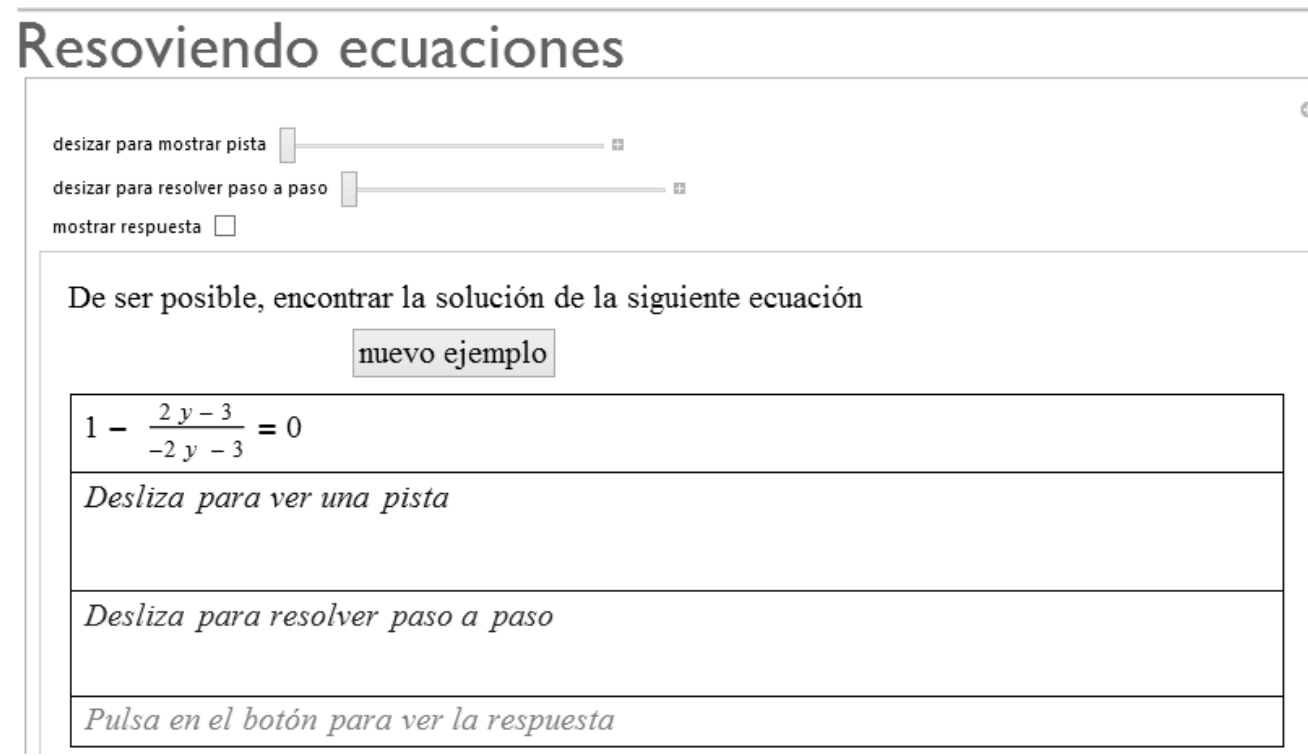

Figura 1. Resolviendo ecuaciones

Como se puede ver, al trabajar con expresiones racionales se le exigió al estudiante repasar conceptos previamente abordados como operaciones, simplificación de expresiones racionales y exclusiones de elementos que no pertenecían al conjunto solución, así como también categorizar el tipo de ecuación que queda al simplificar la expresión.

Para ayudar al estudiante a interiorizar estos procesos, se les dotó de un programa con dos barras de deslizamiento: la primera ayuda al estudiante recordando los pasos vistos en clase al abordar este tipo de ecuaciones, la segunda ayuda al estudiante resolviendo la ecuación paso a paso, ya que la solución se puede realizar de muchas maneras. Este programa le recuerda al estudiante que los pasos mostrados no son la única vía de solución y que es probable resolverla de otro modo. Lo importante es que la respuesta debe ser la misma.

Debajo de las barras de ayuda está la opción de mostrar o no la respuesta, en la figura 2 se muestra el programa con todas las ayudas y la respuesta activa.

El botón "nuevo ejemplo" cambia el ejercicio, el programa está diseñado para que el estudiante repase gran variedad de este tipo de ecuaciones haciendo poco probable que el ejercicio se repita. 


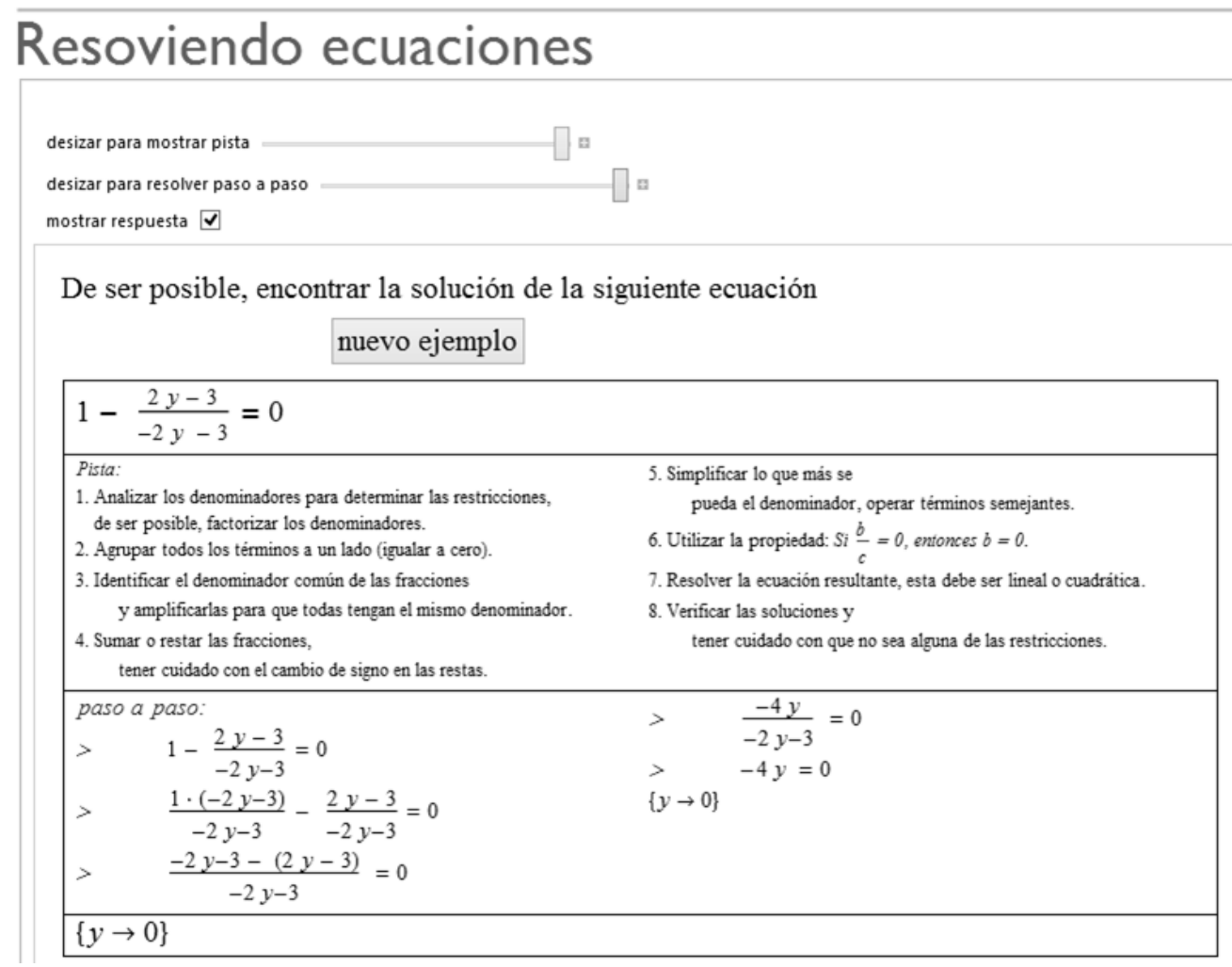

Figura 2. Resolviendo ecuaciones

\subsection{Inecuación no lineal}

De un modo similar al que se utilizó para desarrollar las ecuaciones, se comenzó con el desarrollo de problemas que involucraran el uso de intervalos para hallar la respuesta, pues en este caso pueden ser infinitas soluciones. Se trabajaron problemas lineales y problemas no lineales. La interfaz del usuario es muy similar al de los programas anteriores como se muestra en la figura 3.

Como se muestra en la figura 3, se cuenta con las barras deslizantes que ayudan al estudiante y también se cuenta con la opción de mostrar o no la respuesta. Con este programa se hace especial énfasis en el uso correcto de los intervalos y en su representación gráfica. En la figura 4 se muestra el programa con todas las ayudas y la respuesta activa.

Con este tipo de ejercicios, se refuerza todo el trabajo anterior, pues el estudiante necesita operar expresiones algebraicas y resolver ecuaciones para poder resolver ejercicios.

\subsection{Transformaciones de funciones}

En el tercer corte, se trabajó el concepto de funciones, abordando problemas que impliquen identificar variables y constantes; y modelar, acorde a las características, del problema. Una vez introducido el concepto de función, se trabajó 


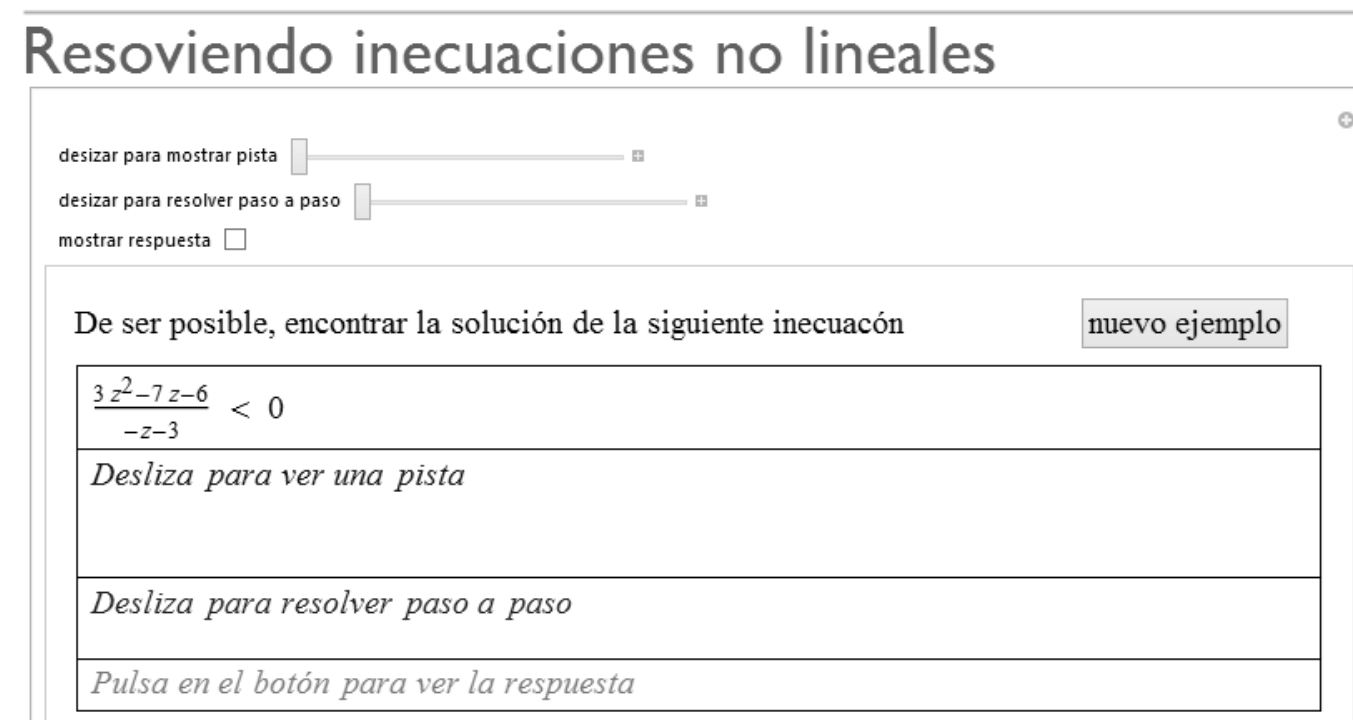

Figura 3. Resolviendo inecuaciones no lineales

\section{Resoviendo inecuaciones no lineales}

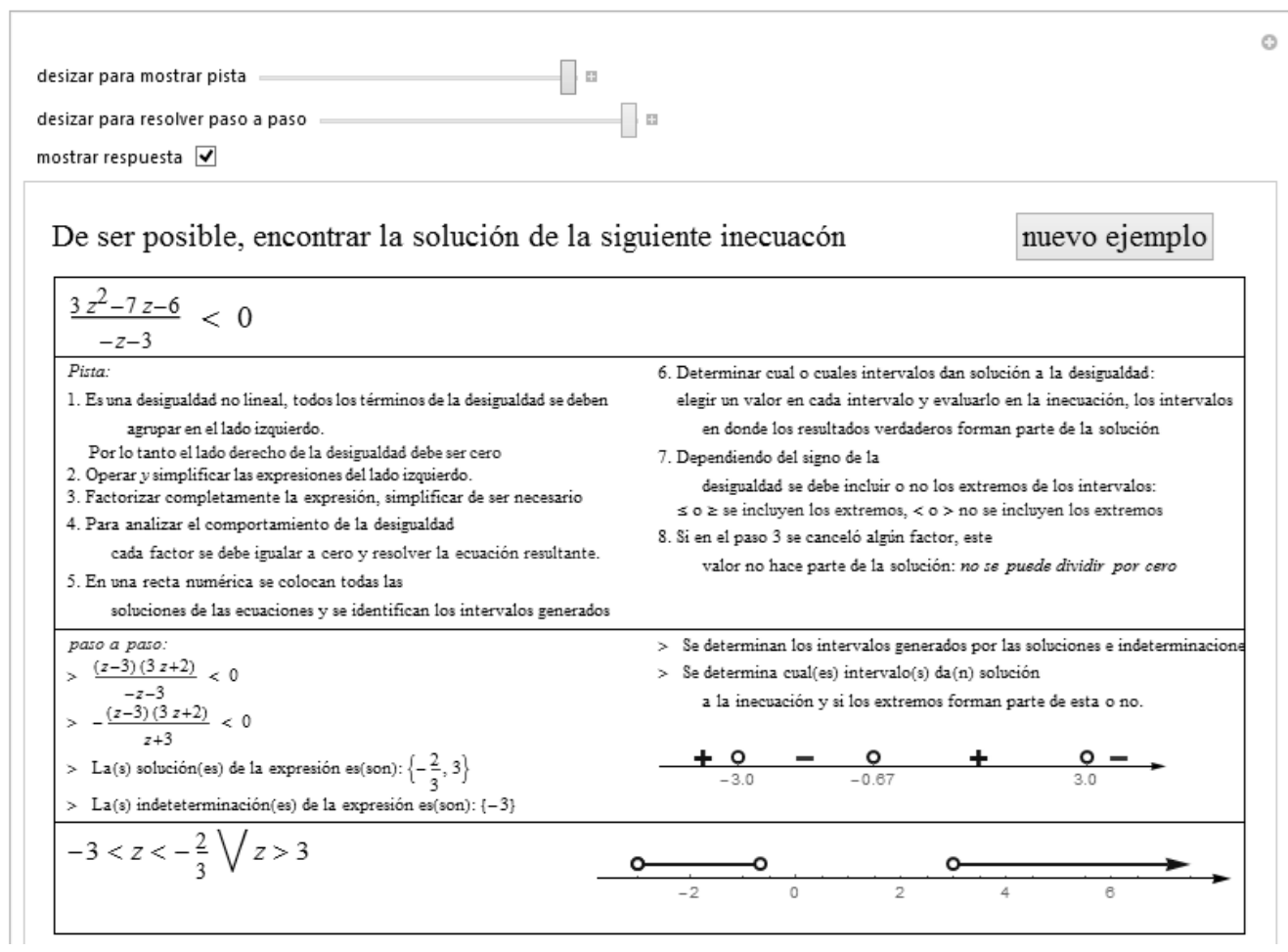

Figura 4. Resolviendo inecuaciones no lineales 
su representación gráfica y específicamente las transformaciones de funciones. En la figura 5 se presenta la interfaz gráfica del programa "transformaciones de funciones".
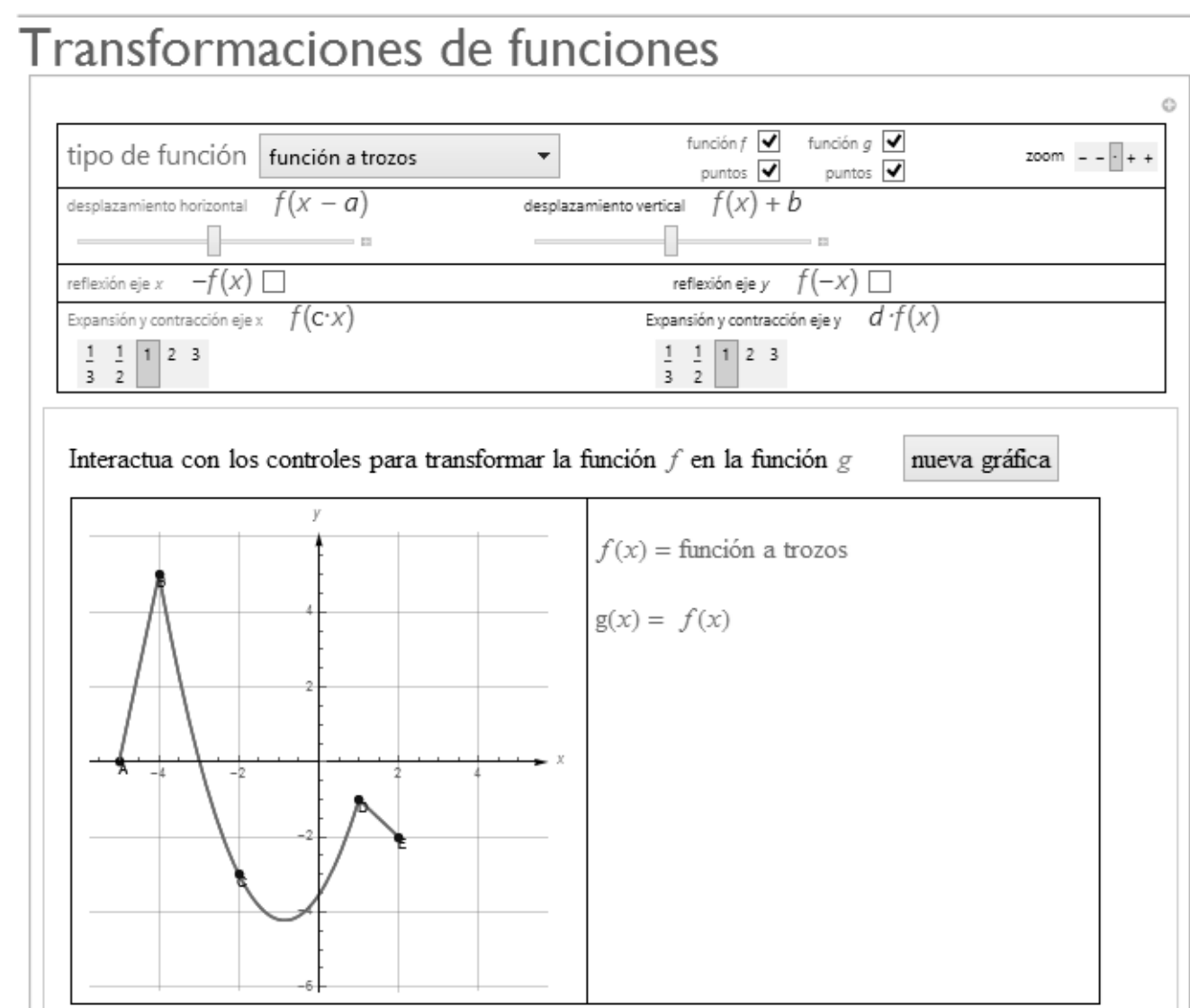

Figura 5. Transformaciones de funciones

Este programa cuenta con una gran cantidad de elementos manipulables, entre ellos: cambiar el tipo de función: puede ser a trozos, una recta, una parábola o una raíz cuadrada. También se puede elegir qué tipo de transformación se realiza, se estudia el desplazamiento vertical y horizontal, la reflexión con respecto al eje $y$ y al eje $x$ y la expansión y contracción con respecto a los ejes cartesianos.

El estudiante puede interactuar a tal punto de juntar todos los tipos de transformaciones para ver la función resultante, tanto gráficamente como analíticamente, como se muestra en la figura 6.

Los controles superiores de la derecha, se utilizan para ocultar las funciones (original y transformada) o los puntos de estas y para modificar el zoom, esto permite que, en clase, este programa se pueda utilizar como un facilitador de ejercicios y repaso de conceptos. 


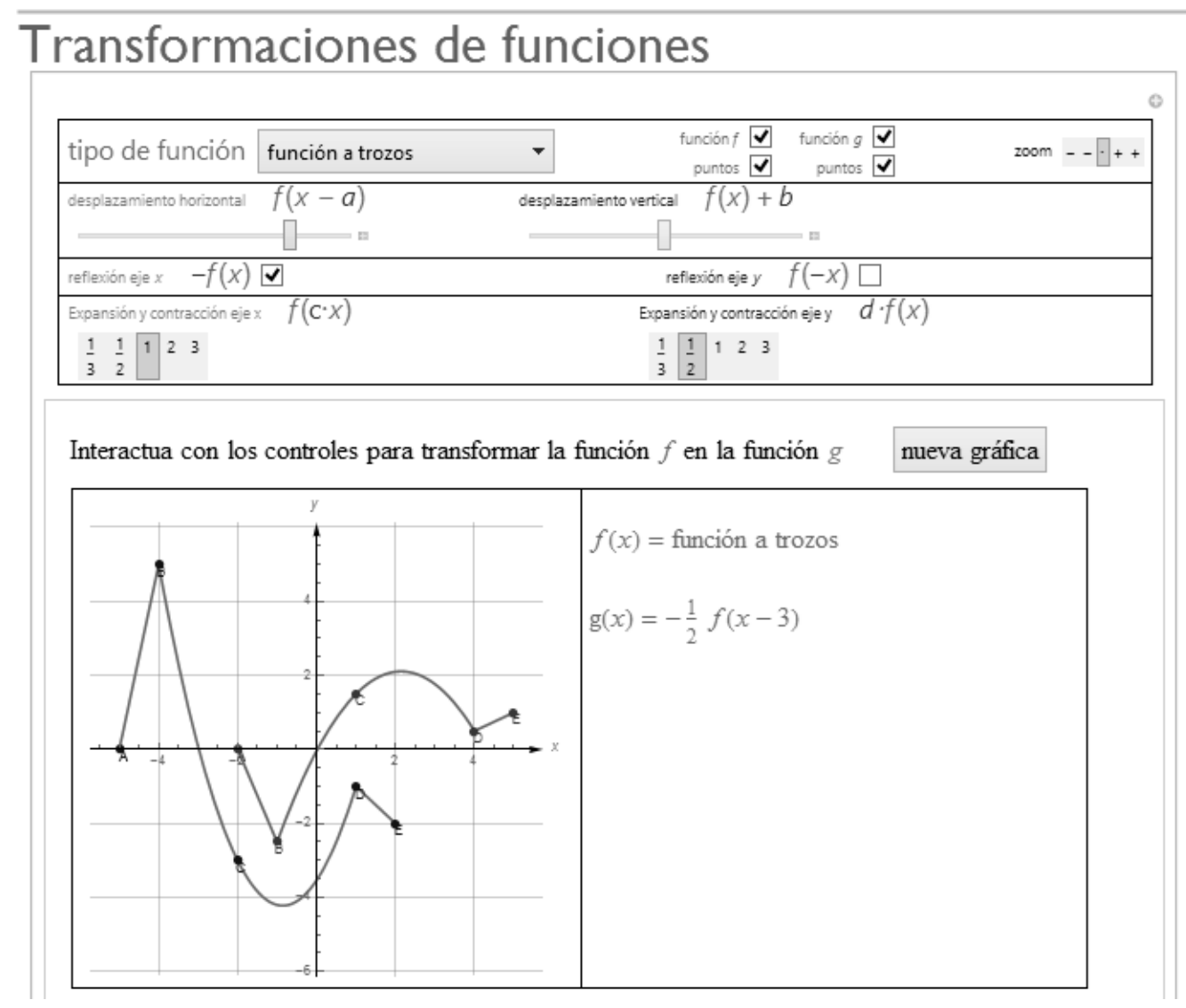

Figura 6. Transformaciones de funciones

\subsection{Función cuadrática}

El último tema abordado en el curso de matemáticas es la función cuadrática, se hace especial énfasis en el trabajo de diferentes representaciones (algebraica, tabular y gráfica).

El objetivo es facilitar que el estudiante identifique las principales características de una función cuadrática como los cortes e interceptos con los ejes, el vértice, la simetría y la concavidad. Como resultado, se espera que dada la expresión algebraica de alguna función cuadrática, se pueda graficar, identificando los principales puntos de esta. En la figura 7 se presenta la interfaz gráfica del programa.

$\mathrm{Al}$ igual que todos los programas, este cuenta con ejemplos de forma aleatoria lo que garantiza que las funciones no se repitan; además se tiene una serie de preguntas para que el estudiante interiorice el concepto de función (no sólo de la función cuadrática) y con una barra deslizadora que responda dichas preguntas. En la figura 8 se muestra el programa con todas las ayudas y las respuestas. 


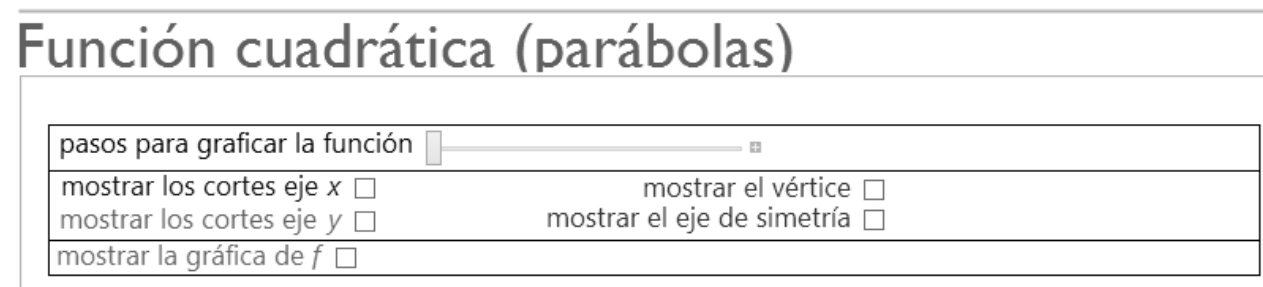

Determine los ceros, corte eje $y$ y vértice de la parábola cuya función es

$$
f(x)=\frac{3 x^{2}}{2}-3 x
$$

nueva función
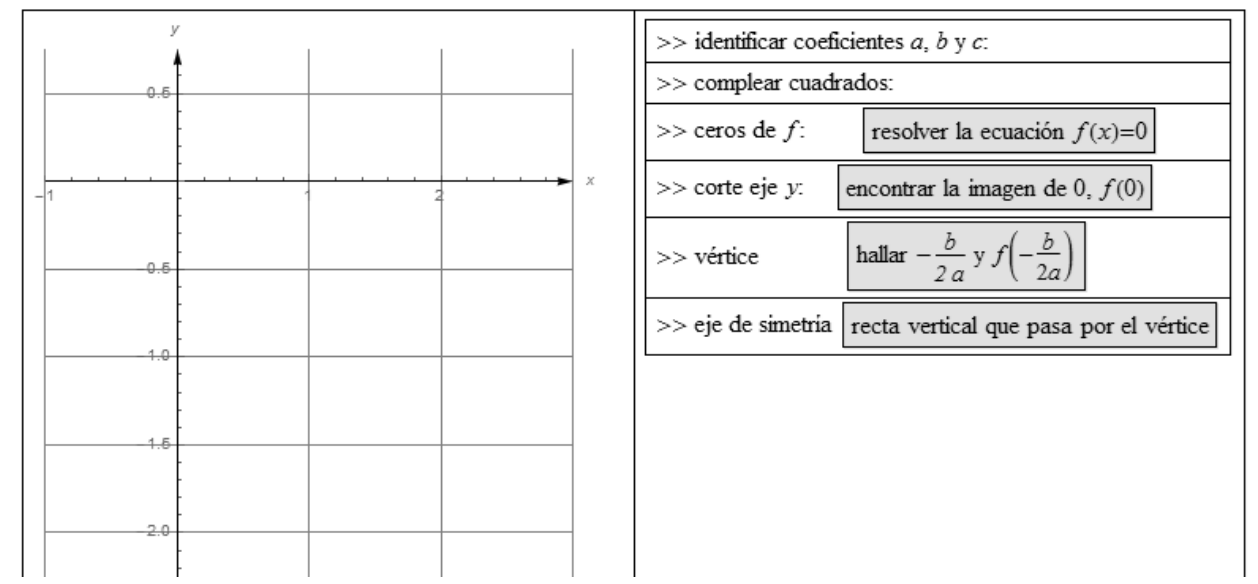

Figura 7. Función cuadrática

Las funciones pueden tener o no ceros, lo cual implica en el estudiante el trabajo de resolver la ecuación (repasar conceptos vistos anteriormente), también pueden ser cóncavas hacia arriba o abajo.

La naturaleza de este programa acerca al estudiante al proceso de graficar funciones, el cual se profundiza en el curso de cálculo.

\subsection{Primera experiencia en el aula}

Al utilizar los programas en el aula se identificaron tres momentos para su uso: en el primero se expone el ejercicio acorde al concepto estudiado (ecuación, inecuación, función, entre otros) sin ningún tipo de ayuda ni respuesta y se brinda un tiempo a los estudiantes para que lo desarrollen, de ser necesario, se ayuda al estudiante que lo requiera. El tiempo de desarrollo depende de la dificultad de los ejercicios.

El segundo momento, se desarrolla cuando se muestra solo la solución de la ecuación y los respectivos mensajes de ayuda, lo cual da tiempo para que aquellos que no llegaron a la solución puedan revisar y corregir los errores.

En el último momento, se muestra el desarrollo paso a paso y se compara con lo propuesto por los estudiantes, se da un tiempo para responder las preguntas 


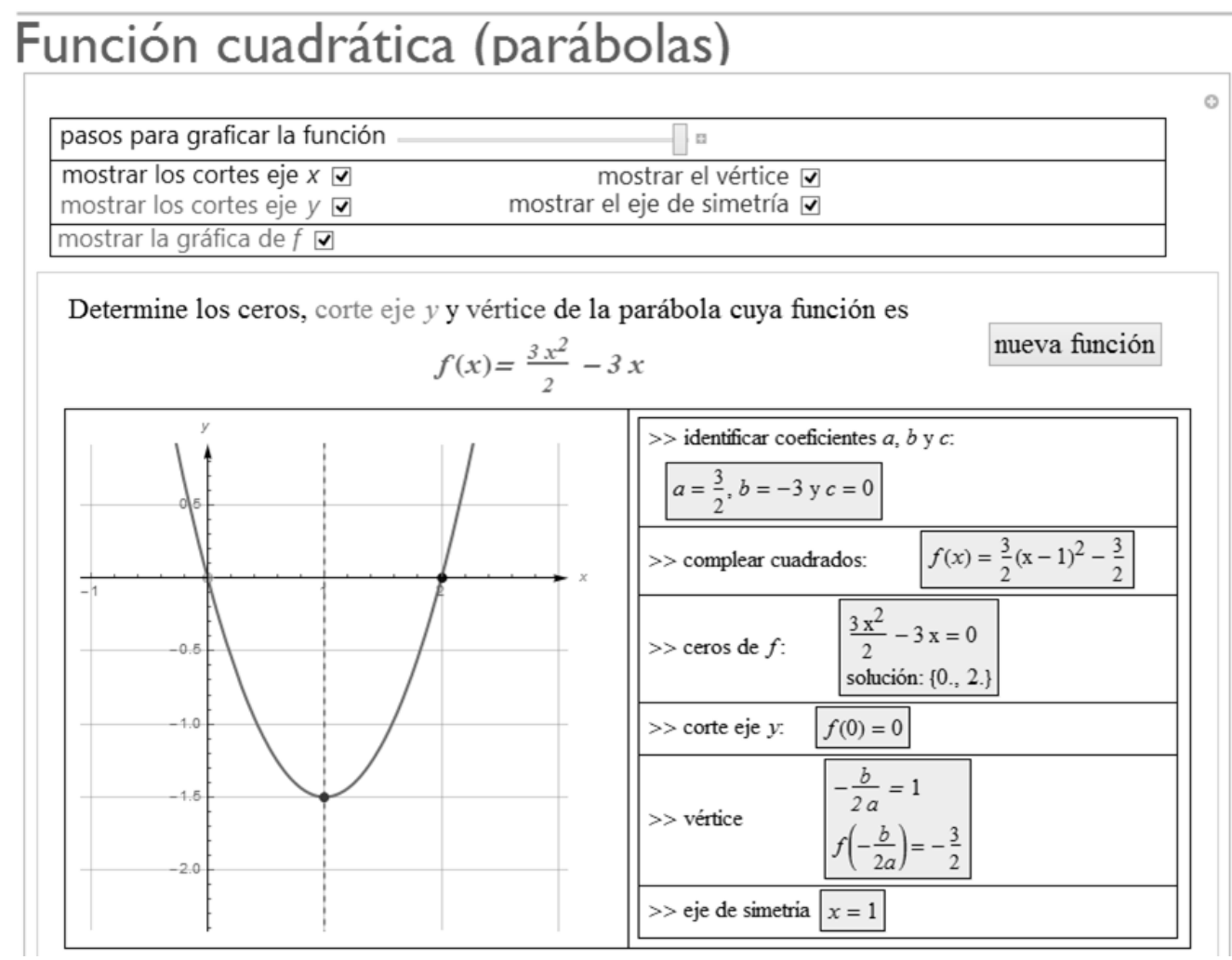

Figura 8. Función cuadrática

que se tenga y el docente está pendiente de aquellos que no lo hicieron de la misma manera para ayudarles a corregir los errores y poder solucionar el ejercicio.

En general, desde que se comenzaron a utilizar estas herramientas en el aula de clase, se vió un cambio en la disposición de la mayoría de los estudiantes, pues se confirmó que el manipularlas o aplicarlas en clase, brindó una mayor seguridad y un constante acompañamiento en los procesos algorítmicos, promoviendo en el estudiante una motivación mayor a trabajar en clase.

$\mathrm{Al}$ comienzo, no se entregaron los programas a los estudiantes, sólo se utilizaron en el aula de clase, pero por requerimiento de ellos a medida que se abordaba un nuevo tema se subía el respectivo programa a la plataforma virtual. El trabajo en casa es algo que aún no se puede medir, pero sí se evidencia que algunos estudiantes descargan las herramientas y las utilizan; incluso algunos en la siguiente clase tienen preguntas específicas del desarrollo de determinado ejercicio.

\section{Discusión y conclusiones}

La naturaleza de estos programas permite potenciar el desarrollo algorítmico de los ejercicios propuestos y estudiados en la clase, y al ser este el fuerte, también 
es su principal debilidad porque sin el pertinente trabajo en clase por parte del profesor, se puede llegar a cometer el grave error de dedicar la mayoría de la clase en procesos algorítmicos y formar estudiantes que sólo desarrollan y refuerzan este tipo de procesos, pero que en ningún momento desarrollan competencias matemáticas o se forman para afrontar situaciones de la vida cotidiana.

El objetivo, por tanto, es poder trabajar solo una parte de la clase en procesos algorítmicos y tener una herramienta que oriente al estudiante en este proceso para poder enfocar el grueso de la clase la solución de problemas y al desarrollo de estudiantes competentes matemáticamente. Lamentablemente esto no se ha logrado en un $100 \%$ pues algunas veces se excede el tiempo dedicado al desarrollo algorítmico de uno o varios ejercicios y no se tiene tiempo para trabajar con situaciones problema.

Al aplicar la experiencia se confirma que la herramienta puede cumplir el propósito planeado, pero que un incorrecto uso puede llevar al error de utilizarla como sustituto de intuiciones y comprensiones conceptuales en el estudiante.

Aunque la implementación de los programas sólo se ha hecho en un curso, indiscutiblemente se ha evidenciado un cambio en la participación y motivación de los estudiantes en el trabajo del aula de clase. El simple hecho de tener la herramienta presente, muestra una disposición diferente al abordar un ejercicio y la metodología de la clase se ve influenciada positivamente.

\section{Síntesis}

La primera conclusión a la que se llega es la siguiente: el programa Mathematica 10, además de ser una herramienta para utilizar en el aula por el docente y los estudiantes como apoyo en la comprensión de los temas trabajados.

La segunda conclusión es: todos los programas cumplen con el objetivo de que el estudiante repase (en clase o en sus momentos de estudio) los conceptos abordados en la materia y entienda los algoritmos y operaciones necesarios para resolver ejercicios algorítmicos.

La experiencia en el aula da cuenta que no se puede estancar todo el tiempo de trabajo en clase o estudio en la resolución de ejercicios algorítmicos, el reto está en utilizar estos para potenciar el desarrollo de problemas aplicados y fomentar un desarrollo de competencias matemáticas y pensamiento de alto nivel en los estudiantes.

\section{Referencias}

1. Cantoral, R. Cordero, Farfan R. Imaz, E: Una revisión de la Investigación Educativa. En Cálculo y Análisis. Universidad Autónoma del Estado de México UAEM. Segundo Simposio Internacional sobre Investigación en Educación Matemática. 1991.

2. Córdoba, J. Vingues, G. Cárdenas, C. Martínez, F. Obando, G. Posada, F. Jiménez, A. Sepúlveda, J: Herramientas computacionales en el desarrollo de procesos de interpretación y argumentación en clase de matemáticas. Ministerio de Educación, 
Memorias del congreso internacional: Tecnologías computacionales en el currículo de Matemáticas. Bogotá, Colombia. 2002.

3. García, G. Serrano, C. Díaz, H: Una aproximación epistemológica, didáctica y cognitiva a nociones básicas y conceptos del Cálculo. Universidad Pedagógica Nacional. 1999.

4. Godino, J. D. Batanero, C. Font, V.: Fundamentos de la Enseñanza y el Aprendizaje de las Matemáticas para Maestros. Granada, Universidad de Granada, 2003.

5. López, O. \& Triana, S. Efecto de un activador computacional de autoeficacia sobre el logro de aprendizaje en estudiantes de diferente estilo cognitivo. Revista Colombiana de Educación, 64. 2013.

6. Meyer, D: Math class needs a makeover. TED talks. 2010.

7. Moreno, L. Santos, M: Proceso de transformación del uso de la tecnología en una herramienta para la solución de problemas de matemáticas por parte de los estudiantes. Seminario Nacional de Formación Docente. Uso de nuevas tecnologías en el aula de clase, Ministerio de educación, serie memorias. 2002.

8. Steward, James. Cálculo: trascendentes tempranas. 6a. ed. México: Cengage Learning, 2008.

9. Wolfram, C: Teaching kids real math with computers. TED talks. 2010. 